\title{
Hadron Spectra from a Non-Relativistic Model with Confining Harmonic Potential
}

(Espectros de Hadrons a partir de um modelo não relativístico com potencial harmônico confinante

\author{
Eduardo Cuervo-Reyes \\ Instituto Superior de Ciencias y Tecnología Nucleares-ISCTN, \\ Ave. Salvador Allende y Luaces, Quinta de los Molinos, La Habana, Cuba \\ Marcos Rigol \\ Institut für Theoretische Physic III, Universität Stuttgart, \\ Pfaffenwaldring 57, D-70550 Stuttgart, Germany \\ Centro Brasileiro de Pesquisas Físicas-CBPF, \\ Rua Dr. Xavier Sigaud 150, 22229-180, Rio de Janeiro, Brazil \\ Jesus Rubayo-Soneira \\ Instituto Superior de Ciencias y Tecnología Nucleares-ISCTN, \\ Ave. Salvador Allende y Luaces, Quinta de los Molinos, La Habana, Cuba \\ Recebido em 25 de julho, 2002. Aceito em 28 de outubro, 2002.
}

\begin{abstract}
Hadron spectra and other properties of quark systems are studied in the framework of a non-relativistic spinindependent phenomenological model. The chosen confining potential is harmonic, which allowed us to obtain analytical solutions for both meson and baryon (of equal constituent quarks) spectra. The introduced parameters are fixed from the low-lying levels of heavy quark mesons. The requirement of flavor independence is imposed, and it restricts the possible choices of inter-quark potentials. The hyper-spherical coordinates are considered for the solution of the three-body problem.
\end{abstract}

Espectros de Hadrons e outras propriedades de sistemas de quarks são estudados do ponto de vista de um modelo fenomenológico não-relativístico independente de spin. O potencial confinante escolhido é harmônico, o qual nos permite obter soluções analíticas tanto para os espectros dos mésons quanto para os dos bárions (de iguais quarks constituintes). Os parâmetros introduzidos são fixos a partir dos primeiros estados excitados dos mésons pesados. A condição de independência de sabor é imposta, o que restringe as possíveis escolhas de potenciais inter-quarks. As coordenadas hiper-esféricas são consideradas para a solução do problema de três corpos.

\section{Introduction}

The study of the fundamental or constituent blocks of matter has been for long time a fascinating field in physics. With the pass of the years new fundamental blocks or particles have appeared, modifying old concepts. For example the atom, that was initially supposed to be fundamental, was found to be formed by the nucleus and electrons, the nucleus was found to be formed by neutrons and protons (nucleons) and finally the nucleons to be formed by quarks.

Nowadays it is believed that the theory describing the interactions between fundamental particles is the Standard Model. This model has a $S U(3) \times S U(2) \times U(1)$ sym- metry. The $S U$ (3) component is what is called Quantum Chromodynamics (QCD) and it is the gauge field theory describing the strong interactions of quarks and gluons. The $S U(2) \times U(1)$ component is called Standard Electroweak Model describing interactions between quarks and leptons through the $W$ bosons and the photons. There is an additional particle in the electroweak model, the neutral Higgs scalar that appears after the spontaneously symmetry breaking mechanism, whose existence has not been probed experimentally and that is a subject of a lot of present experimental research because it could prove the validity of the theory.

We will be interested in the present paper in the low 
energy region of the QCD theory, in which quarks interact strongly to form bound states known as hadrons. When these bound states are formed by a quark and an antiquark $(q \bar{q})$ they are called mesons; and when they are formed by 3 -quark states $(q q q)$ they are called baryons. The study of the hadron spectra is a fundamental and open field in theoretical physics. Up to the moment the more important approaches to this subject have been: Lattice QCD, QCD sum rules and potential models. Lattice QCD is the most fundamental approach and together with QCD sum rules have had good success. Potential models, although less fundamentals, have proved to be very useful even in non-relativistic approximations. Since the latest seventies a lot of attempts have been made in this field with very good results [1].

An important question for the use of potential models is whether it is possible to consider hadrons as non-relativistic bound states of quarks. This question could be answered solving the Schrodinger equation assuming a $q \bar{q}$ potential and then checking if the obtained quark velocities are nonrelativistic. For the charmonium system this was done in Ref.[2] and it was obtained a result of $\left\langle v^{2} / c^{2}\right\rangle=0.2$ in the ground state. For lighter hadrons the results are no so good and the validity of the non-relativistic approximation depends strongly from the interaction potential chosen. For example, the interaction potential proposed by De Rujula, Georgi and Glashow [3] was shown [4] to be unsuitable for dynamical non-relativistic calculations of light hadrons. However, Martin showed latter [5, 6, 7] that a non-relativistic model with a power law potential is able to describe heavy quark mesons and the clearly relativistic $s \bar{s}$ states. The potential proposed by Martin had the form

$$
V(r)=A+B r^{\alpha},
$$

where $A=-8.093 \mathrm{GeV}, B=6.898 \mathrm{GeV}$ and $\alpha=0.1$.

The study of baryon systems with this power law potential was done by Richard [8] obtaining good results. At that time the baryon spectra in potential models, although rather elaborated [9], was completely disconnected from the meson sector. Exceptions were some attempts to derive meson and baryon potential energies from a common framework as the instanton, string or bag model. The rule adopted by Richard for the $q q$ potential was

$$
V_{q q}=\frac{1}{2} V_{q \bar{q}}
$$

Other very well known phenomenological nonrelativistic potential models have been based in the Cornel potential $[10,2]$

$$
V(r)=A_{C} r-\frac{B_{C}}{r}+C_{C},
$$

where $A_{C}=0.1756 \mathrm{GeV}^{2}, B_{C}=0.52$ and $C_{C}=$ $-0.8578 \mathrm{GeV}$; and the logarithmic potential [11]

$$
V(r)=A_{L}+B_{L} \ln (r),
$$

where $A_{L}=-0.6631 \mathrm{GeV}$ and $B_{L}=0.733 \mathrm{GeV}$.
Finally, other two propositions of inter-quark potentials are due to Song and Ling [12]

$$
V(r)=A_{S} r^{\frac{1}{2}}+B_{S} r^{-\frac{1}{2}}
$$

where $A_{S}=0.511 \mathrm{GeV}^{\frac{3}{2}}$ and $B_{S}=0.923 \mathrm{GeV}^{\frac{1}{2}}$; and Turin [13]

$$
V(r)=-A_{T} r^{-\frac{3}{4}}+B_{T} r^{\frac{3}{4}}+C_{T} .
$$

where $A_{T}=0.620 \mathrm{GeV}^{\frac{1}{4}}, B_{T}=0.304 \mathrm{GeV}^{\frac{7}{4}}$ and $C_{T}=-0.823 \mathrm{GeV}$.

Studies with phenomenological potentials, like the Cornell one, but considering a relativistic kinetic energy term, are able to describe the observed spectra of heavy and light hadron systems $[14,15]$. Non-trivial connections between these relativistic potential models and rigorous numerical results from lattice QCD have been demonstrated [16]. Some recent works have tried to understand why the non-relativistic treatment works and allows useful predictions even for relativistic systems [17, 18].

In the previously mentioned potential models, central conditions have been the flavor independence of the potential chosen and the existence of a confining term. Quark masses appearing in these phenomenological models are the so called constituent quark masses that should not be confused with the current quark masses, that are the mass parameters appearing in the QCD Lagrangian. Constituent quark masses are bigger than current quark masses and it is suppose that this is due to gluonic condensate effects. In general constituent quark masses are considered as free parameters to fit in potential models, that is why different values are found all over the literature. Up to our knowledge there are two works in which constituent quark masses are calculated from QCD: one is due to Elias et al. [19, 20] that used an operator-product expansion (OPE) of approximate non-perturbative vacuum expectation values in the fixedpoint gauge; the other work is due to Cabo and Rigol [21] in which a Modified Perturbative QCD expansion incorporating gluon condensation was employed [22]. Because there are no free quarks, a lot of care should be taken with the meaning of these quark masses.

In the present work we study the hadron spectra within a non-relativistic spin-independent phenomenological model, with a harmonic confining potential. The idea is to show with an educational perspective what can be done with the Schrodinger equation, the very useful for physicists harmonic potential, and the same number of parameters used in almost all the calculations, in order to understand the hadron spectroscopy. The harmonic potential has the great advantage that allowed us to obtain analytical solutions for both the meson and baryon (with equal constituent quarks) spectra. Although the obtained results are not as good as the ones obtained for the former potentials, we think that they give very good estimates of the hadron properties without the need of numerical calculations implemented for all the previously mentioned potentials. As we will work with a spin independent model, the magnitudes we will deal with 
will be spin averaged. For fitting our parameters spin averages of experimental values were calculated, but for certain resonances the experimental values were not at hand [23] and theoretical results obtained by Fulcher [24] were used. It should be mentioned that in a work by Hirata et al. [25] the harmonic oscillator was employed as an unperturbed confinement potential, in the asymptotically free coloredquark-gluon model in which the one-gluon-exchange force was treated perturbatively. Later Ram and Hasala [26] used the pure harmonic oscillator potential in the Klein-Gordon equation to determine the meson masses.

The exposition will be organized as follows. In Section II meson spectra is calculated for two different potentials with harmonic confining terms, that allowed to obtain analytical solutions. The first potential is the harmonic oscillator and the second one is the harmonic oscillator plus a term proportional to $\frac{1}{r^{2}}$. The results are analyzed for the flavor dependent and independent cases. In Section III we study the properties of the radial wave function, and some related physical quantities, at the origin. Section IV is devoted to the study of the baryon spectra and the summary can be found in Section 5. Finally, an Appendix was intro- duced for mathematical details of the three-body problem solution.

\section{Meson Spectra}

In the present section, the non-relativistic meson spectra are calculated for two different potentials. The first one is the pure harmonic potential and in the second one a term proportional to $\frac{1}{r^{2}}$ is added to the harmonic oscillator, allowing to improve the short-range interaction. That is the potentials considered are:

$$
V_{1}(r)=\frac{k_{1} r^{2}}{2}+W_{1}
$$

and

$$
V_{2}(r)=\frac{k_{2} r^{2}}{2}-\frac{\alpha}{r^{2}}+W_{2}
$$

The Schrodinger equation, in the Center-of-Mass (CM) system and in spherical coordinates, has the form (notation $\hbar=c=1$ is considered)

$$
\left\{-\frac{1}{2 \mu}\left\{\frac{1}{r^{2}} \frac{\partial}{\partial r}\left(r^{2} \frac{\partial}{\partial r}\right)+\frac{1}{r^{2}}\left[\frac{1}{\sin \theta} \frac{\partial}{\partial \theta}\left(\sin \theta \frac{\partial}{\partial \theta}\right)+\frac{1}{\sin ^{2} \theta} \frac{\partial^{2}}{\partial \phi^{2}}\right]\right\}-\left(E_{i}-V_{i}\right)\right\} \Psi_{i}(r, \theta, \phi)=0,
$$

where $i=1,2$ and $\mu=\frac{m_{1} m_{2}}{m_{1}+m_{2}}$ is the reduced mass. Introducing the spherical harmonics

$$
\Psi_{i, n l_{i} m_{i}}(r, \theta, \phi)=\frac{1}{r} R_{i, n l_{i}}(r) Y_{i, l_{i} m_{i}}(\theta, \phi),
$$

the radial Schrodinger equation can be written as

$$
\left\{-\frac{1}{2 \mu} \frac{d^{2}}{d r^{2}}+\frac{k_{i} r^{2}}{2}+\frac{l_{i}\left(l_{i}+1\right)}{2 \mu r^{2}}-\left(E_{i, n l_{i}}-W_{i}\right)\right\} R_{i, n l_{i}}(r)=0,
$$

where

$$
l_{i}\left(l_{i}+1\right)=\left\{\begin{array}{c}
l(l+1) \text { for } i=1 \\
l(l+1)-2 \mu \alpha \text { for } i=2 .
\end{array}\right.
$$

The solution of Eq. (11) can be found in any classical textbook of Quantum Mechanics [27, 28] and has the form

$$
R_{i, n l_{i}}(\xi)=N_{i, n l_{i}} e^{\frac{-\xi^{2}}{2}} \xi^{l_{i}+1} F\left(-n, l_{i}+\frac{3}{2}, \xi^{2}\right),
$$

where

$$
\xi=\frac{r}{\sqrt{\frac{1}{\sqrt{\mu k}}}}
$$

$N_{i, n l_{i}}$ is a normalization factor and $F\left(-n, l_{i}+\frac{3}{2}, \xi^{2}\right)$ is the confluent hyper-geometric function. The eigenvalues of the energy are

$$
E_{i}=\sqrt{\frac{k}{\mu}}\left(2 n+l_{i}+\frac{3}{2}\right)+W_{i}
$$

First, in order to compare with experimental values we fitted the parameters without imposing the flavor independence condition on the potentials. For that case [5] it is only possible to determine from the experimental spectra the values of $\varepsilon=\sqrt{\frac{k}{\mu}}, 2 \mu \alpha$ and $V_{0}=W_{0}+m_{1}+m_{2}$ that are shown in Table 1 for charmonium $(c \bar{c})$ and upsilon $(b \bar{b})$ systems. As it can be seen, the parameter $2 \mu \alpha$ has the limiting value for the existence of the solution for the flavor dependent (FD) potential $V_{2}$ for both quark systems, because

$$
l_{2}=-\frac{1}{2}+\sqrt{\left(l+\frac{1}{2}\right)^{2}-2 \mu \alpha}
$$

and for $l=0$ and $2 \mu \alpha>0.25$ the squared root will have imaginary values. 
Table 1: Parameters obtained for the $c \bar{c}$ and $b \bar{b}$ systems with the flavor dependent potential.

\begin{tabular}{||c|c|c|c||}
\hline \hline System & $\varepsilon(M e V)$ & $2 \mu \alpha$ & $V_{0}=W_{0}+m_{1}+m_{2}(\mathrm{MeV})$ \\
\hline \hline$V_{1}^{F D 1}(c \bar{c})$ & 296 & & 2679 \\
$V_{2}^{F D 2}(c \bar{c})$ & 302 & 0.25 & 2773 \\
$V_{1}^{F D}(b \bar{b})$ & 217 & & 9235 \\
$V_{2}^{F D}(b \bar{b})$ & 219 & 0.25 & 9301 \\
\hline \hline
\end{tabular}
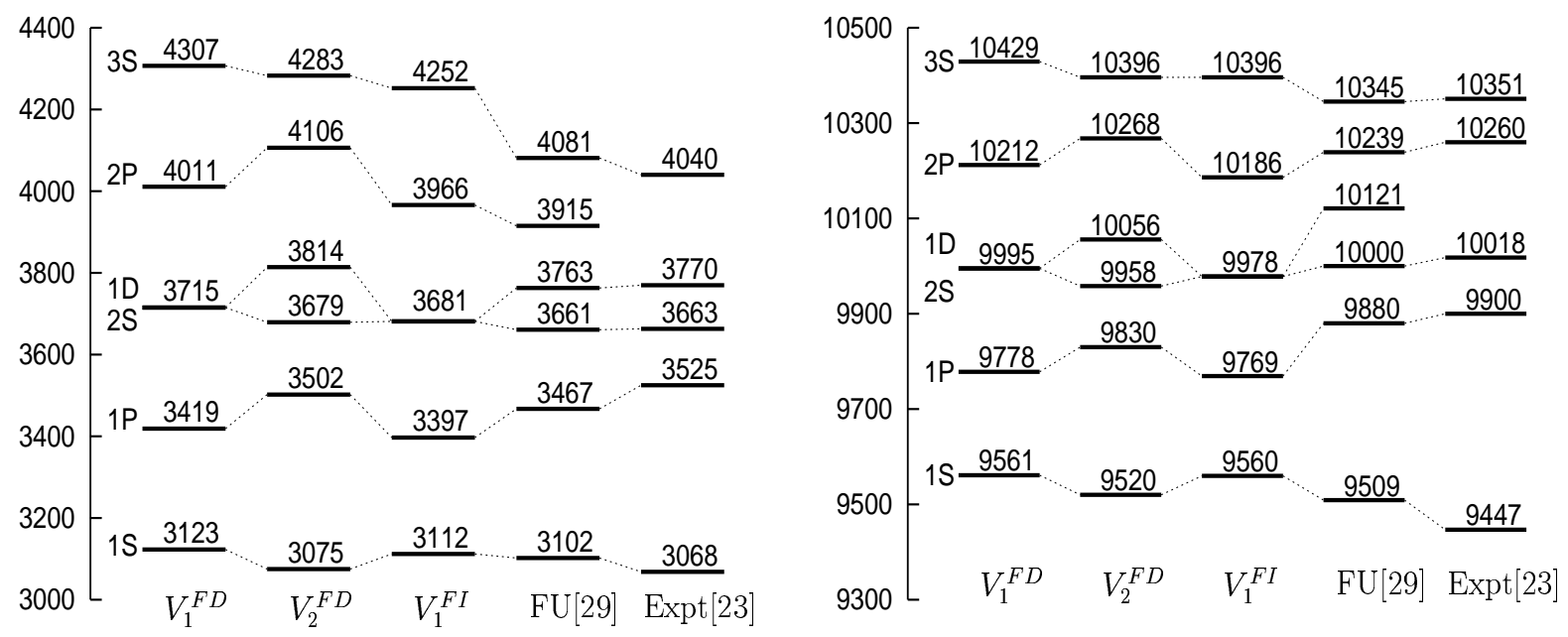

Figure 1: Charmonium (left) and Upsilom (right) energies $(\mathrm{MeV})\left(V_{1}^{F I}\right.$ is the flavor independent potential $\left.V_{1}(r)\right)$.

The mass values obtained for $c \bar{c}$ and $b \bar{b}$ systems, with the parameters in Table 1, are presented in Figure 1 together with the theoretical values obtained by Fulcher [29] and compared with the experimental values [23] (the results for each potential are presented in the figure as columns). The experimental masses of the states $1 S, 1 P, 2 S$ for the charmonium systems and all the ones presented for the Upsilom systems are spin averaged and were used in our fit of the parameters. The experimental masses reported for the $1 D$ and $3 S$ charmonium resonances belong to the $1^{3} D_{1}$ and $3^{3} S_{1}$ states. The results obtained for the potential $V_{2}$ are in better agreement with the experimental results because the non-harmonic term $\left(\sim \frac{1}{r^{2}}\right)$ improves the behavior of the potential in the region $r \rightarrow 0$, and breaks some degeneracy present in the solution of the pure harmonic oscillator. Unfortunately the potential $V_{2}$ was found incompatible with the flavor independence condition, been impossible to obtain a unique potential like this for charmonium and upsilon systems. Then, after requiring the flavor independence condition only the pure harmonic oscillator parameters can be fitted, and the following values were obtained

$$
\begin{array}{cl}
k=0.155 \mathrm{GeV}^{3}, & m_{s}=2.725 \mathrm{GeV}, \\
W_{0}=-4.94 \mathrm{GeV}, & m_{c}=3.812 \mathrm{GeV}, \\
& m_{b}=7.093 \mathrm{GeV},
\end{array}
$$

where the $s$ quark was included as in Martin's works. The spectra calculated with these parameters, for $c \bar{c}$ and $b \bar{b}$ mesons are presented in Figure $1\left(V_{1}^{F I}\right)$. Other meson resonances like $s \bar{s}, c \bar{s}-s \bar{c}, b \bar{s}$ and $b \bar{c}$ are shown in Table 2 . The mass of the $1 S$ resonance for the $s \bar{s}$ meson was employed to fit the $s$ quark mass.

As it can be seen the theoretical results obtained are only estimates for the experimental values. The main differences between these theoretical results and the experimental ones, or other theoretical calculations, are clearly due to the nonsingularity of the potential at the origin, and its concavity. That is, the harmonic potential has positive second derivative at variance with other proposed potentials that vary more slowly with the distance. This causes that when the energy of a state increases, the classical allowed region will be smaller for the harmonic potential than for the other ones and states result more localized. Other undesirable effects are the constant spacing between the consecutive levels and 
Table 2: Other meson resonances $(\mathrm{MeV})$.

\begin{tabular}{||c|c|c|c|c|c|c|c|c|c||}
\hline \hline State & $V_{1}^{F I}(s \bar{s})$ & $\operatorname{Expt}^{1}(s \bar{s})$ & $V_{1}^{F I}(c \bar{s})$ & $\operatorname{Expt}^{1}(c \bar{s})$ & $V_{1}^{F I}(s \bar{b})$ & $\operatorname{Expt}^{1}(s \bar{b})$ & $V_{1}^{F I}(b \bar{c})$ & $\operatorname{Expt}^{1}(b \bar{c})$ & $\mathrm{FU}^{2}(b \bar{c})$ \\
\hline \hline $1 \mathrm{~S}$ & 1016 & 1019 & 2065 & 2076 & 5299 & 5370 & 6340 & 6400 & 6361 \\
$1 \mathrm{P}$ & 1353 & & 2377 & & 5580 & & 6590 & & 6703 \\
$2 \mathrm{~S}$ & 1690 & & 2689 & & 5861 & & 6840 & & 6876 \\
\hline \hline
\end{tabular}

the degeneracy present. The differences between the proposed potential and the former ones also cause the so called constituent quarks masses to be here like twice of the usual values for the heavy quarks $c, b$ and fifth times for the $s$ quarks. The addition of the non harmonic term improved the results for the lower levels and broke the degeneracy present, but for the higher excited states the deficiencies remain; and this potential was also unable to fit with the flavor independence condition.

Two interesting magnitudes to evaluate, related with the wave functions at the origin, are the leptonic widths and the hyperfine splitting.

Leptonic widths for charmonium and upsilon systems are presented in Figure 3 and compared with results in Ref. $[33,24]$. They were obtained by the formula [35]

$$
\Gamma\left(V^{0} \rightarrow e^{+} e^{-}\right)=\frac{16 \pi N_{c} \alpha^{2} e_{q}^{2}}{3} \frac{|\Psi(0)|^{2}}{M_{V}^{2}},
$$

where $N_{c}=3$ (number of colors), $\alpha$ denotes the fine structure constant, $e_{q}$ denotes the quark charge and $M_{V}$ is the mass of the vector meson.

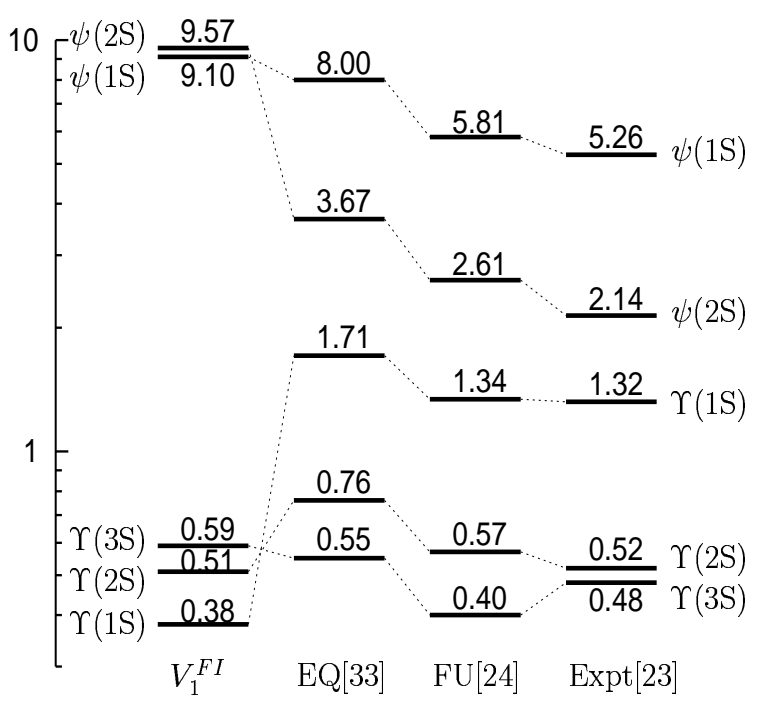

Figure 3: Leptonic widths $(\mathrm{KeV})$.

Finally, the hyperfine splitting can be obtained through the expression [5]

Figure 2: Radial wave functions at the origin and related quantities $\left(\left|R_{n l}^{(l)}(0)\right|^{2}(G e V)^{2 l+3}\right)$ for $c \bar{b}$ mesons.

$$
M\left({ }^{3} S_{1}\right)-M\left({ }^{1} S_{0}\right)=C t e \frac{|\Psi(0)|^{2}}{m_{a} m_{b}},
$$


in which the constant is fixed through the hyperfine splitting observed in the charmonium family

$$
M(J / \psi)-M\left(\eta_{c}\right)=117 M e V .
$$

Results for the $c \bar{c}\left(J / \psi={ }^{3} S_{1} \eta_{c}={ }^{1} S_{0}\right), b \bar{c}\left(B_{c}^{*}={ }^{3} S_{1}\right.$ $\left.B_{c}={ }^{1} S_{0}\right)$ and $b \bar{b}\left(\Upsilon={ }^{3} S_{1} \eta={ }^{1} S_{0}\right)$ resonances are shown in Figure 4.

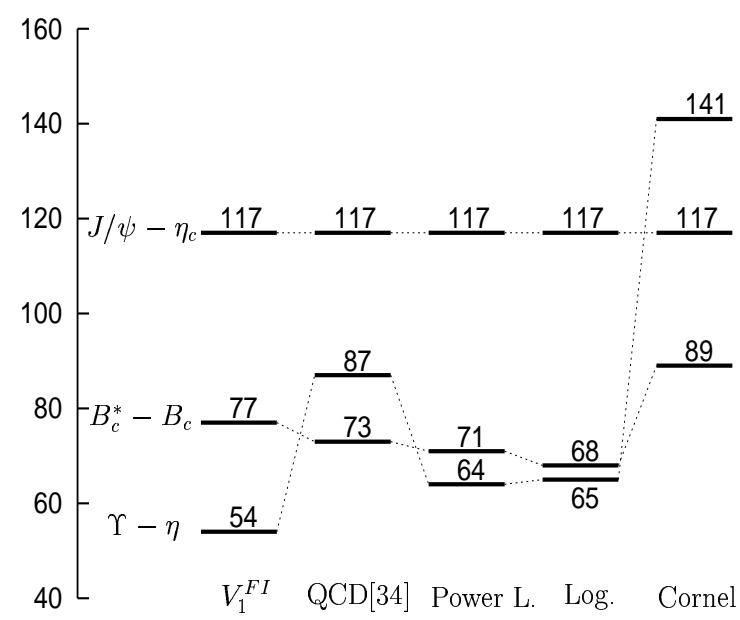

Figure 4: Hyperfine splitting for quarkonium ground states $(\mathrm{MeV})$.

\section{Baryon Spectra}

In the present section we study the baryon spectra using the harmonic potential obtained in Section II. For the threebody system the Hamiltonian has the form

$$
\begin{aligned}
H & =-\frac{1}{2 m_{1}} \nabla_{r_{1}}^{2}-\frac{1}{2 m_{2}} \nabla_{r_{2}}^{2}-\frac{1}{2 m_{3}} \nabla_{r_{3}}^{2} \\
& +V_{12}\left(r_{12}\right)+V_{23}\left(r_{23}\right)+V_{31}\left(r_{31}\right) .
\end{aligned}
$$

In order to separate the C-M motion, we define the Jacobi $(\vec{r}, \vec{R})$ and C-M $\left(\vec{R}_{C M}\right)$ coordinates through

$$
\begin{aligned}
\vec{r} & =\left[\frac{\mu_{b c}}{\mu_{a, b c}}\right]^{\frac{1}{4}}\left(\overrightarrow{r_{b}}-\overrightarrow{r_{c}}\right), \\
\vec{R} & =\left[\frac{\mu_{a, b c}}{\mu_{b c}}\right]^{\frac{1}{4}}\left(\overrightarrow{r_{a}}-\frac{m_{b} \overrightarrow{r_{b}}+m_{c} \overrightarrow{r_{c}}}{m_{b}+m_{c}}\right), \\
\vec{R}_{C M} & =\frac{m_{a} \overrightarrow{r_{a}}+m_{b} \overrightarrow{r_{b}}+m_{c} \overrightarrow{r_{c}}}{M},
\end{aligned}
$$

where

$$
\begin{aligned}
\mu_{b c} & =\frac{m_{b} m_{c}}{m_{b}+m_{c}}, \\
\mu_{a, b c} & =\frac{m_{a}\left(m_{b}+m_{c}\right)}{M}, \\
M & =m_{a}+m_{b}+m_{c} .
\end{aligned}
$$

Considering equal masses $m_{a}=m_{b}=m_{c} \equiv m$, and Eqs. (18), (19) the C-M motion is separated and the Hamiltonian for the relative motion takes the form

$$
H=-\frac{1}{2 \mu}\left(\nabla_{r}^{2}+\nabla_{R}^{2}\right)+\frac{\sqrt{3}}{4} k\left(\vec{r}^{2}+\vec{R}^{2}\right)+\frac{3}{2} W_{0},
$$

with

$$
\mu \equiv\left(\frac{m_{a} m_{b} m_{c}}{M}\right)^{\frac{1}{2}}=\frac{m}{\sqrt{3}},
$$

where the rule adopted by Richard [8] (2), was considered.

At this point we could obtain the baryon spectra directly from Eq. (20) noticing that it is the sum of two independent harmonic oscillators. But then the energy eigenvalues will not be in terms of the natural quantum numbers of the system, and it will not be possible a check with experimental or other theoretical results. The same problem is faced with the usual 3-dimensional harmonic oscillator, which could be solved in Cartesian coordinates as the sum of three independent 1-dimensional harmonic oscillators, but then no relation between the conserved angular momentum and the energy spectrum is obtained and the use of spherical coordinates is convenient.

Then introducing the hyper-spherical coordinates [36] (see Appendix A)

$$
\begin{aligned}
r_{x} & =\rho \cos (\chi) \sin \left(\theta_{r}\right) \cos \left(\varphi_{r}\right), \\
r_{y} & =\rho \cos (\chi) \sin \left(\theta_{r}\right) \sin \left(\varphi_{r}\right), \\
r_{z} & =\rho \cos (\chi) \cos \left(\theta_{r}\right), \\
R_{x} & =\rho \sin (\chi) \sin \left(\theta_{R}\right) \cos \left(\varphi_{R}\right), \\
R_{y} & =\rho \sin (\chi) \sin \left(\theta_{R}\right) \sin \left(\varphi_{R}\right), \\
R_{z} & =\rho \sin (\chi) \cos \left(\theta_{R}\right),
\end{aligned}
$$

the kinetic term stay in a diagonal form and the potential become only dependent of the hyper-radio, then the Hamiltonian (20) takes the form

$$
\begin{aligned}
H= & -\frac{1}{2 \mu}\left[\frac{1}{\rho^{5}} \frac{\partial}{\partial \rho}\left(\rho^{5} \frac{\partial}{\partial \rho}\right)+\frac{1}{\rho^{2}}\left(\frac{1}{\sin ^{2}(2 \chi)} \frac{\partial}{\partial \chi}\left(\sin ^{2}(2 \chi) \frac{\partial}{\partial \chi}\right)+\frac{\widehat{J}^{2}\left(\theta_{r}, \varphi_{r}\right)}{\cos ^{2}(\chi)}+\frac{\widehat{L}^{2}\left(\theta_{R}, \varphi_{R}\right)}{\sin ^{2}(\chi)}\right)\right] \\
& +\frac{\sqrt{3}}{4} k\left(\rho^{2}\right)+\frac{3}{2} W_{0},
\end{aligned}
$$

In which $\widehat{J}$ is the angular momentum of the subsystem $b c$ and $\widehat{L}$ is the angular momentum of particle $a$ respect to the C-M of 
the two body subsystem $b c$.

$$
\begin{aligned}
\widehat{J}^{2} & =-\frac{1}{\sin \left(\theta_{r}\right)} \frac{\partial}{\partial \theta_{r}}\left(\sin \theta_{r} \frac{\partial}{\partial \theta_{r}}\right)-\frac{1}{\sin ^{2}\left(\theta_{r}\right)} \frac{\partial^{2}}{\partial \varphi_{r}} \\
\widehat{L}^{2} & =-\frac{1}{\sin \left(\theta_{R}\right)} \frac{\partial}{\partial \theta_{R}}\left(\sin \theta_{R} \frac{\partial}{\partial \theta_{R}}\right)-\frac{1}{\sin ^{2}\left(\theta_{R}\right)} \frac{\partial^{2}}{\partial \varphi_{R}} .
\end{aligned}
$$

The Schrodinger equation in this case has also analytical solution (see Appendix A), with eigenvectors

$$
\begin{aligned}
\Psi_{N, \lambda, j, m_{j}, l, l_{j}}\left(\xi, \chi, \theta_{r}, \varphi_{r}, \theta_{R}, \varphi_{R}\right)= & N_{N, \lambda, j, l} e^{-\frac{\xi^{2}}{2}} \xi^{\lambda} L_{N}^{\lambda+2}\left(\xi^{2}\right) \cos ^{j+\frac{1}{2}}(\chi) \sin ^{l+\frac{1}{2}}(\chi) \times \\
& \times P_{\frac{\lambda-j-l}{2}}^{l+\frac{1}{2}, j+\frac{1}{2}}(\cos (2 \chi)) Y_{l}^{m_{l}}\left(\theta_{R}, \varphi_{R}\right) Y_{j}^{m_{j}}\left(\theta_{r}, \varphi_{r}\right),
\end{aligned}
$$

where

$$
\lambda=2 n+j+l .
$$

The eigenvalues are given by the expression

$$
E=\sqrt{\frac{\sqrt{3}}{2}} \sqrt{\frac{k}{\mu}}(2 N+\lambda+3)+\frac{3}{2} W_{0},
$$

where $N$ is the number of nodes of the hyper-radial function, $\lambda$ is the grand-angular quantum number, and $P_{\frac{\lambda-j-l}{2}}^{l+\frac{1}{2}, j+\frac{1}{2}}(\cos (2 \chi))$ are the Jacobi polynomials. In Figures 5 and 6 we compare our results with other calculations presented by Richard in Ref [8].

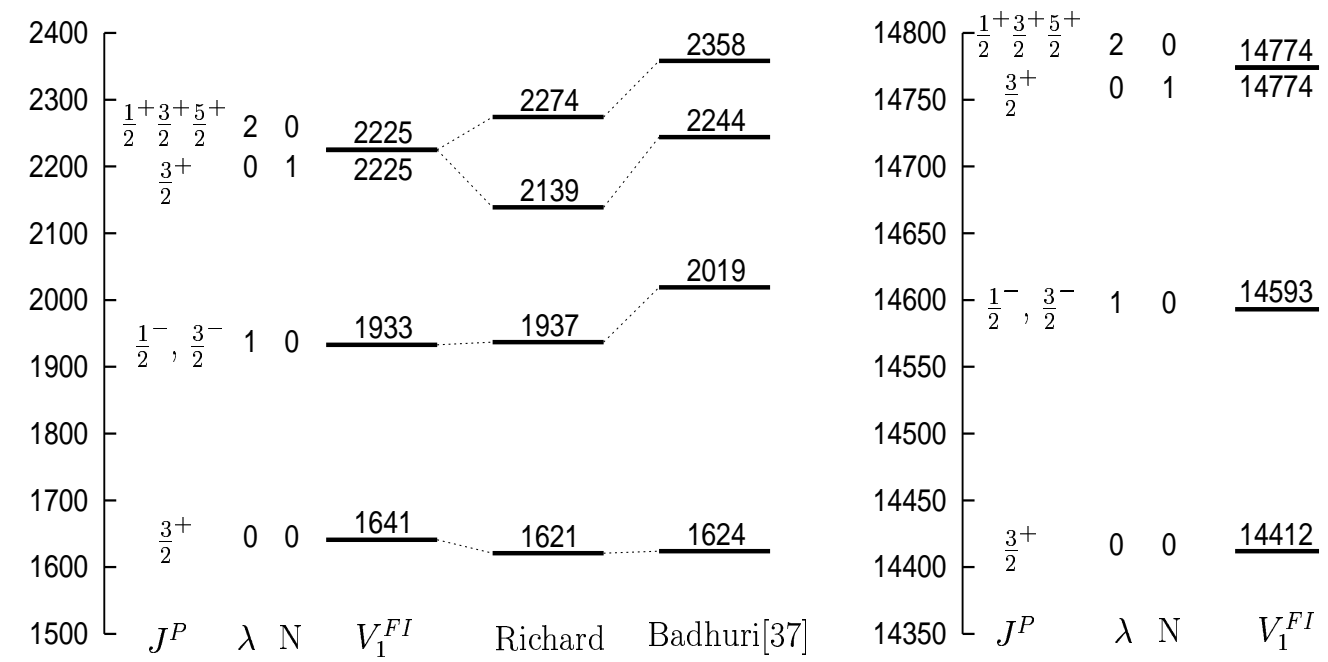

Figure 6: Baryon sss (left) and $b b b$ (right) energies $(\mathrm{MeV})$.

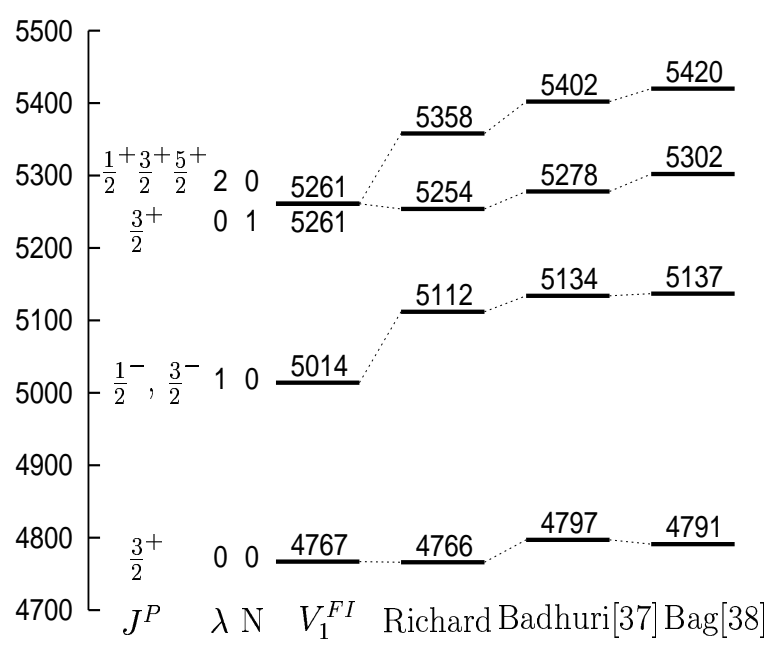

Figure 5: Baryon $c c c$ energies $(\mathrm{MeV})$. 
To compare with experiments there were only two equal constituent quark baryons at hand [30], for the sss system, for which

$$
\begin{gathered}
m\left(\Omega^{-}\right)_{E x p t}=2250 \mathrm{MeV}, \\
m\left(\Omega^{-}\right)_{V_{1}^{F I}}=2225 \mathrm{MeV}, \\
\frac{m\left(\Omega^{-}\right)_{E x p t}-m\left(\Omega^{-}\right)_{V_{1}^{F I}}}{m\left(\Omega^{-}\right)_{E x p t}}=0.01 .
\end{gathered}
$$

\section{Conclusions}

In the present paper we have studied, within a nonrelativistic spin-independent model with harmonic confining potential, the spectra and other properties of hadron systems.

It was found that for mesons, without imposing the flavor independence condition, two possible potentials with harmonic confining terms had analytical solutions that give good estimates of the experimental values reported for the meson spectra. The better fit was obtained for the potential with a term proportional to $\frac{1}{r^{2}}$ because it has a singularity for $r \rightarrow 0$ that improves its behavior in this region. However this potential was found to be incompatible with the flavor independence condition and was not considered in the analysis that followed.

For the pure harmonic oscillator the parameters introduced were fixed from the low lying levels of heavy quarks systems and imposing the flavor independence condition. The calculation of the meson and baryon spectra, and the hyperfine splitting with this potential give good estimates of the experimental and other theoretical results; in the case of leptonic widths we could say that the results are not good. Although this potential is far from being a good approximation for the real inter-quark potential, and the results are not as good as the theoretically obtained by other phenomenological models, it has the great advantage that allows to obtain analytical solutions for both meson and baryon spectra. That is, reasonable theoretical results are obtained without the need of numerical methods and computational calculations. The major differences of this potential and the others mentioned in the introduction are due to its non-singularity at the origin and its concavity, that cause the bad results for the obtained leptonic widths and also (for a better fit with experiments) the so called constituent masses to be bigger than the usual ones. The baryon spectra was studied with the use of the rule (2) for the $q q$ potential and for obtaining the analytical solution it was necessary to restrict the study to equal constituent quark systems.

We finalize with the conclusions of A. Martin in Ref. [7] "... if you want to know the mass of a particle and if you have a little time (in years!) and little money you should forget all your prejudices and use potential models".

\section{A The hyper-spherical coordinates and the solution of the three-body problem with a harmonic potential}

The hyper-spherical coordinates are very useful for dealing with the three-body problem; in what follows we make a small review of them.

The kinetic energy of the Hamiltonian (20),

$$
\widehat{K}=-\frac{1}{2 \mu}\left(\nabla_{r}^{2}+\nabla_{R}^{2}\right)
$$

can be written as a Laplacian in a 6-dimensional space, due to the symmetry in the two Jacobi vectors. When a change of coordinates is made, the $N$-dimensional Laplacian transforms as

$$
\Delta=\frac{1}{\prod_{i} l_{i}} \sum_{k=1}^{N} \frac{\partial}{\partial x_{k}^{\prime}}\left(\frac{\prod_{j} l_{j}}{l_{k}^{2}} \frac{\partial}{\partial x_{k}^{\prime}}\right) \text { where } l_{j}=\sqrt{\sum_{i=1}^{N}\left(\frac{\partial x_{i}}{\partial x_{j}^{\prime}}\right)^{2}},
$$

are the metric coefficients. The change to hyper-spherical coordinates is based on the definition of the hyper-radius by

$$
\rho=\sqrt{\sum_{i=1}^{N} x_{i}^{2}},
$$

and $N-1$ angles in a way that (29) is satisfied and the old variables are expressed in terms of the new ones by $N$ functions with the form

$$
x_{i}=\rho F_{i}\left(\Omega_{N-1}\right)
$$


and the Laplacian operator becomes

$$
\Delta=\frac{1}{\rho^{N-1}} \frac{\partial}{\partial \rho}\left(\rho^{N-1} \frac{\partial}{\partial \rho}\right)+\frac{1}{\rho^{2}} \Delta_{\Omega_{N-1}} .
$$

In the expression (31) $\Omega_{N-1}$ denotes all angles. The explicit form of the angular term of the Laplacian operator and its eigenfunctions will depend of the set of angles selected as new coordinates and the eigenvalues will be equal to $-\lambda(\lambda+N-2)$. In this 6-dimensional case where the selected coordinates are (21) the angular term obtained is

$$
\Delta_{\Omega_{N-1}}=\frac{1}{\sin ^{2}(2 \chi)} \frac{\partial}{\partial \chi}\left(\sin ^{2}(2 \chi) \frac{\partial}{\partial \chi}\right)+\frac{1}{\cos ^{2}(\chi)} \Delta_{\theta_{r}, \varphi_{r}}+\frac{1}{\sin ^{2}(\chi)} \Delta_{\theta_{R}, \varphi_{R}} .
$$

Its eigenfunctions are expressed as a product of orthogonal polynomials in separated variables [36]. The eigenfunctions corresponding to Jacobi's angles $\theta_{r}, \varphi_{r}, \theta_{R}$ and $\varphi_{R}$ are the well known spherical harmonics. Then the equation obtained for the function of the angle $\chi$ is

$$
\left[\frac{1}{\sin ^{2}(2 \chi)} \frac{\partial}{\partial \chi}\left(\sin ^{2} 2 \chi \frac{\partial}{\partial \chi}\right)-\frac{l(l+1)}{\sin ^{2}(\chi)}-\frac{j(j+1)}{\cos ^{2}(\chi)}\right] F_{\lambda}^{l, j}(\chi)=-\lambda(\lambda+4) F_{\lambda}^{l, j}(\chi),
$$

for which the solution are the Jacobi polynomials

$$
F_{\lambda}^{l, j}(\chi)=N_{\lambda, l, j} \sin ^{l+\frac{1}{2}}(\chi) \cos ^{j+\frac{1}{2}}(\chi) P_{\frac{\lambda-j-l}{2}}^{l+\frac{1}{2}, j+\frac{1}{2}}(\cos (2 \chi)),
$$

with $\lambda=2 n+l+j$ and $N_{\lambda, l, j}$ a normalization factor.

For the harmonic interaction between equal mass particles, the potential is only dependent of the hyper-radius, then we can separate variables and the radial equation has the form

$$
\left[-\frac{1}{2 \mu \rho^{5}} \frac{\partial}{\partial \rho}\left(\rho^{5} \frac{\partial}{\partial \rho}\right)+\frac{\lambda(\lambda+4)}{2 \mu \rho^{2}}+\frac{\sqrt{3} k}{4} \rho^{2}-\left(E_{N, \lambda}-\frac{3}{2} W_{0}\right)\right] R_{N, \lambda}(\rho)=0 .
$$

Introducing new variables

$$
\xi=\left(\frac{\rho}{\rho_{0}}\right) \sqrt{\beta}, \quad \rho_{0}=\left(\frac{1}{2 \mu\left(E-\frac{3}{2} W_{0}\right)}\right)^{\frac{1}{2}}, \quad \beta^{2}=\frac{(k \sqrt{3})}{8 \mu\left(E-\frac{3}{2} W_{0}\right)^{2}}
$$

and the new function

$$
T_{N, \lambda}=\frac{R_{N, \lambda}}{\xi^{\frac{5}{2}}}
$$

we obtain for (35) the equation

$$
\left[\frac{d^{2}}{d \xi^{2}}+\frac{1}{\beta_{N, \lambda}}-\frac{\lambda(\lambda+4)+\frac{15}{4}}{\xi^{2}}-\xi^{2}\right] T_{N, \lambda}(\xi)=0
$$

with solutions

$$
T_{N, \lambda}(\xi)=e^{-\frac{\xi^{2}}{2}} \xi^{\lambda+\frac{5}{2}} L_{N}^{\lambda+2}\left(\xi^{2}\right),
$$

where

$$
\frac{1}{\beta_{N, \lambda}}=4 s+2(\lambda+2)+2
$$

and $L_{N}^{\lambda+2}\left(\xi^{2}\right)$ are the generalized Laguerre polynomials.

Then the complete eigenvectors and the eigenvalues of the problem are given by Eqs. (24) and (26).

\section{Acknowledgments}

One of us (M.R.) would like to thank A. Dimarco, S. Duarte, and the Department of Nuclear and High Energy Physics at "Centro Brasileiro de Pesquisas Físicas (CBPF)" for their kind hospitality and useful discussions.

\section{References}

[1] E. Eichten, Talk given at the "Third International Conference at Vanderbilt University on New Results in High Energy Physics", Nashville, Tennessee, March 6-8 (1978).

[2] E. Eichten, K. Gottfried, T. Kinoshita, K. D. Lane and T. M. Yan, Phys. Rev. D 21, 203 (1980). 
[3] A. DeRujula, H. Georgi and S. L. Glashow, Phys. Rev. D 12, 147 (1975).

[4] R. K. Bhaduri, L. E. Cohler and Y. Nogami, Phys. Rev. Lett. 44, 1369 (1980).

[5] A. Martin, Phys. Lett. B 93, 338 (1980); B 100, 511 (1988).

[6] A. Martin, Phys. Lett. B 214, 561 (1980).

[7] For a recent summary; A. Martin, Concluding Remarks of the Workshop "Quark Confinement and the Hadron Spectrum II", edited by N. Brambilla and G. Prosperi, Wold Scientific, Singapure (1997).

[8] J. M. Richard, Phys. Lett. B 100, 515 (1981).

[9] Proceedings of "Baryon 80", edited by N. Isgur and G. Karl, University of Toronto.

[10] E. Eichten, K. Gottfried, T. Kinoshita, K. D. Lane and T. M. Yan, Phys. Rev. D 17, 3090 (1978); Erratum-ibid. D 21, 313 (1980).

[11] C. Quigg and J. L. Rosner, Phys. Lett. B 71, 153 (1977).

[12] X. Song and H. Lin, Z. Phys. C34, 223 (1987).

[13] D. B. Lichtenberg, E. Predazzi, R. Roncaglia, M. Rosso and J. G. Wills, Z. Phys. C 41, 615 (1989).

[14] A. Gara, B. Durand, L. Durand and L. J. Nickisch, Phys. Rev. D40, 843 (1989).

[15] L. P. Fulcher, Z. Chen and K. C. Yeong, Phys. Rev. D47, 4122 (1993).

[16] A. Duncan, E. Eichten and H. Tacker, Phys. Lett. B303, 109 (1993).

[17] W. Lucha, H. Rupprecht and F. F. Schoberl, Phys. Rev. D46, 1088 (1992).

[18] G. Jaczko and L. Durand, Phys. Rev. D58, 114017 (1998).

[19] V. Elias, T. G. Steele and M. Scadron, Phys. Rev. D38, 1584 (1988).

[20] V. Elias and T. G. Steele, Phys. Lett. B212, 88 (1988).
[21] A. Cabo and M. Rigol, Eur. Phys. J. C23, 289 (2002).

[22] M. Rigol and A. Cabo, Phys. Rev. D62, 074018 (2000).

[23] R. M. Barnett et al., Review of Particle Physics. Particle Data Group, Phys. Rev. D54, 1 (1996); C. Caso et al., Review of Particle Physics. Particle Data Group, Eur. Phys. J. C3, 1 (1998).

[24] L. P. Fulcher, Phys. Rev. D60, 074006 (1999).

[25] K. Hirata, T. Kobayashi and N. Nakamaru, Phys. Rev. D18, 834 (1978).

[26] B. Ram and R. Hasala, Phys. Rev. D19, 3467 (1979).

[27] L. D. Landau and E. M. Lifshits, Mecánica Cuántica NoRelativista, Reverté S.A., Barcelona (1972).

[28] A. S. Davydov, Quantum Mechanics, Pergamon Press, Oxford, $2^{\text {nd }}$ Edition (1976).

[29] L. P. Fulcher, Phys. Rev. D62, 094505 (2000).

[30] D. E. Groom et al., Review of Particle Physics. Particle Data Group, Eur. Phys. J. C15, 1 (2000).

[31] E. Braaten, K. Cheung and T. C. Yuang, Phys. Rev. D48, 5049 (1993).

[32] K. Cheung, Phys. Rev. Lett. 71, 3143 (1993).

[33] E. Eichten and C. Quigg, Phys. Rev. D49, 5845 (1994).

[34] W. Buchmuller and S. H. H. Tye, Phys. Rev. D24, 132 (1981).

[35] R. V. Royen and V. F. Weisskopf, Nuovo Cim. A50, 617 (1967); Erratum-ibid A51, 583 (1967).

[36] Darío deFazio, "Tratazione quantomecanica delle reazione chimiche elementari mediante tecniche Hiperesferiche", Ph.D. Thesis, Perugia University, Italy (1996).

[37] R. K. Bhaduri, L. E. Cohler and Y. Nogami, Print-80-0753 (McMaster) (1980).

[38] P. Hasenfratz, R. R. Horgan, J. Kuti and J. M. Richard, Phys. Lett. B 94, 401 (1980); B95, 299 (1980). 